\title{
ENTIRE DIRICHLET SERIES WITH MONOTONOUS COEFFICIENTS AND LOGARITHMIC h-MEASURE
}

\author{
S.I. Panchuk ${ }^{1}$, T.M. Salo ${ }^{\S}$, O.B. Skaskiv ${ }^{3}$ \\ ${ }^{1,3}$ Department of Mechanics and Mathematics \\ Ivan Franko National University of L'viv \\ L'viv, UKRAINE \\ ${ }^{2}$ Institute of Applied Mathematics and Fundamental Sciences \\ National University "Lvivska Politekhnika" \\ UKRAINE
}

\begin{abstract}
Let $F$ be an entire function represented by absolutely convergent for all $z \in \mathbb{C}$ Dirichlet series of the form $F(z)=\sum_{n=0}^{+\infty} a_{n} e^{z \lambda_{n}}$, where a sequence $\left(\lambda_{n}\right)$ such that $\lambda_{n} \in \mathbb{R}$ and $(\forall n \geq 0): 0 \leq \lambda_{n}<\beta:=\sup \left\{\lambda_{j}: j \geq 0\right\} \leq+\infty$. In this paper we find the condition such that the relation $F(x+i y)=(1+o(1)) a_{\nu(x, F)} e^{(x+i y) \lambda_{\nu(x, F)}}$ holds as $x \rightarrow+\infty$ outside some set $E$ of finite logarithmic $h$-measure (i.e. h-log-meas $(E):=\int_{E \cap[1,+\infty)} h(x) d \ln x<+\infty$ ) uniformly in $y \in \mathbb{R}$, where $h$ is non-decrease positive continuous function on $[0,+\infty)$.
\end{abstract}

AMS Subject Classification: 30B20, 30D20

Key Words: entire Dirichlet series, h-measure, exceptional set

\section{Introduction}

Let $\mathcal{L}$ be the class of positive continuous increasing functions on $[0 ;+\infty)$ and $\mathcal{L}_{+}$the subclass of functions $\Phi \in \mathcal{L}$ such that $\Phi(t) \rightarrow+\infty(t \rightarrow+\infty)$. Вy $\varphi$ we denote inverse function to $\Phi \in \mathcal{L}$.

Received: $\quad$ December 13, 2016

Revised: January 29, 2017

Published: $\quad$ February 28, 2017

${ }^{\S}$ Correspondence author (c) 2017 Academic Publications, Ltd. url: www.acadpubl.eu 
Let $\mathcal{D}$ be a class of entire (absolutely convergent in the whole complex plane $\mathbb{C})$ Dirichlet series of the form

$$
F(z)=\sum_{n=0}^{+\infty} a_{n} e^{z \lambda_{n}}, \quad z \in \mathbb{C},
$$

where a sequence $\left(\lambda_{n}\right)$ such that $\lambda_{n} \in \mathbb{R} \quad(n \geq 0), \lambda_{n} \neq \lambda_{k}$ for any $n \neq k$ and

$$
(\forall n \geq 0): \quad 0 \leq \lambda_{n}<\beta:=\sup \left\{\lambda_{j}: j \geq 0\right\} \leq+\infty .
$$

For $F \in \mathcal{D}$ and $x \in \mathbb{R}$ we denote $M(x, F)=\sup \{|F(x+i y)|: y \in \mathbb{R}\}, m(x, F)=$ $\inf \{|F(x+i y)|: y \in \mathbb{R}\}$, and by $\mu(x, F)=\max \left\{\left|a_{n}\right| e^{x \lambda_{n}}: n \geq 0\right\}, \nu(x, F)=$ $\max \left\{n:\left|a_{n}\right| e^{x \lambda_{n}}=\mu(x, F)\right\}$ the maximal term and central index of series (1), respectively.

By $\mathcal{D}_{a}$ we denote a subclass of Dirichlet series $F \in \mathcal{D}$ with a fixed sequence $a=\left(\left|a_{n}\right|\right),\left|a_{n}\right| \searrow 0\left(n_{0} \leq n \rightarrow+\infty\right)$, and for $\Phi \in \mathcal{L}$ by $\mathcal{D}_{a}(\Phi)$ subclass of functions $F \in \mathcal{D}_{a}$ such that $\ln \mu(x, F) \geq x \Phi(x)\left(x \geq x_{0}\right)$. Let $\mu_{n}:=-\ln \left|a_{n}\right|(n \geq 0)$.

In paper [1] one can find such theorem:

Theorem A (O.B. Skaskiv, 1994 [1]). For every entire function $F \in \mathcal{D}_{a}$ relation

$$
F(x+i y)=(1+o(1)) a_{\nu(x, F)} e^{(x+i y) \lambda_{\nu(x, F)}}
$$

holds as $x \rightarrow+\infty$ outside some set $E$ of finite logarithmic measure, i.e.

$$
\log -\operatorname{meas}(E):=\int_{E} d \ln x<+\infty,
$$

uniformly in $y \in \mathbb{R}$, if and only if

$$
\sum_{n=n_{0}}^{+\infty} \frac{1}{\mu_{n+1}-\mu_{n}}<+\infty .
$$

It is easy to see that relation (3) holds for $x \rightarrow+\infty(x \notin E)$ uniformly in $y \in \mathbb{R}$, if and only if $M(x, F) \sim \mu(x, F)(x \rightarrow+\infty, x \notin E)$, hence it follows $M(x, F) \sim m(x, F)(x \rightarrow+\infty, x \notin E)$.

The finiteness of logarithmic measure of an exceptional set $E$ in Theorem A is the sharp estimate. It follows from such theorem.

Theorem B (Ya.Z. Stasyuk, 2008 [2]). For every increasing sequence $\left(\mu_{n}\right)$, such that condition (4) satisfies and for any function $h \in \mathcal{L}_{+}$there exist an entire Dirichlet series $F \in \mathcal{D}_{a}$ with $\left|a_{n}\right|=\exp \left\{-\mu_{n}\right\}$, a set $E$ and a constant $d>0$ such that h-log-meas $(E):=\int_{E \cap[1,+\infty)} h(x) d \ln x=+\infty$ and $(\forall x \in$ $E): M(x, F) \geq(1+d) \mu(x, F), M(x, F) \geq(1+d) m(x, F)$. 
Due to Theorem B the natural question arises: what conditions must satisfy the entire Dirihlet series $F \in \mathcal{D}_{a}$ in order to relation (3) holds for $x \rightarrow$ $+\infty$ outside some set $E$ of finite logarithmic h-measure, i.e. h- $\log$-meas $(E)<$ $+\infty$ ? In this article we give the answer to the question. Our main result is the following.

Theorem 1. Let $\left(\mu_{n}\right)$ be a sequence such that condition (4) holds, $h \in$ $\mathcal{L}_{+}, \Phi \in \mathcal{L}$ and $F \in \mathcal{D}_{a}(\Phi)$. If

$$
(\forall b>0): \sum_{n=n_{0}}^{+\infty} h\left(\varphi\left(\lambda_{n}\right) \cdot\left(1+\frac{b}{\mu_{n+1}-\mu_{n}}\right)\right) \frac{1}{\mu_{n+1}-\mu_{n}}<+\infty,
$$

then the relation (3) holds as $x \rightarrow+\infty$ outside some set $E$ of finite logarithmic $h$-measure uniformly in $y \in \mathbb{R}$.

The method of proof of Theorem 1 differs from the method of proofs corresponding statements in $[1,3,4,5,6]$ and is close to the methods of proofs from papers $[7,8]$.

\section{Proof of Theorem 1}

We denote $\Delta_{0}=0$ and for $n \geq 1$

$$
\Delta_{n}:=\delta \cdot \sum_{j=0}^{n-1}\left(\mu_{j+1}-\mu_{j}\right) \sum_{m=j+1}^{+\infty}\left(\frac{1}{\mu_{m}-\mu_{m-1}}+\frac{1}{\mu_{m+1}-\mu_{m}}\right), \quad \delta>0 .
$$

It is easy to see that

$$
\Delta_{n} \geq n \delta \quad(n \geq 0), \quad \Delta_{n}=o\left(\mu_{n}\right) \quad(n \rightarrow+\infty) .
$$

We put $b_{n}=e^{\lambda_{n}}$ and consider the Dirichlet series

$$
f(s)=\sum_{n=0}^{+\infty} b_{n} e^{s \mu_{n}}, \quad \mu_{n}=-\ln \left|a_{n}\right| .
$$

The condition $\sum_{n=0}^{+\infty} 1 /\left(\mu_{n+1}-\mu_{n}\right)<+\infty$ implies $n^{2}=o\left(\mu_{n}\right)(n \rightarrow+\infty)$ (see $[1,3])$, thus $\ln n=o\left(\mu_{n}\right)(n \rightarrow+\infty) . F \in \mathcal{D}_{a}$ so $\lim _{n \rightarrow+\infty} \frac{-\ln \left|a_{n}\right|}{\lambda_{n}}=+\infty$. Therefore by Valiron's formula for the abscissa of absolute convergence ([9, p.11]) we get

$$
\sigma_{\mathrm{abs}}(f)=\varliminf_{n \rightarrow+\infty} \frac{-\ln b_{n}}{\mu_{n}}=\lim _{n \rightarrow+\infty}\left(\frac{\ln \left|a_{n}\right|}{\lambda_{n}}\right)^{-1}=0 .
$$

Now we consider Dirichlet series 


$$
f_{q}(s)=\sum_{n=0}^{+\infty} \frac{b_{n}}{\alpha_{n}^{q}} e^{s \mu_{n}}, \quad \alpha_{n}=e^{\Delta_{n}}, q \in \mathbb{R} .
$$

From the second relation in $(6)$ and the condition $\sigma_{\text {abs }}(f)=0$ it follows that

$$
\sigma_{\mathrm{abs}}\left(f_{q}\right)=\varliminf_{n \rightarrow+\infty} \frac{-\ln b_{n}+q \Delta_{n}}{\mu_{n}}=\sigma_{\mathrm{abs}}(f)+q \cdot \lim _{n \rightarrow+\infty} \frac{\Delta_{n}}{\mu_{n}}=0
$$

for any $q \in \mathbb{R}$. Therefore the Dirichlet series of the form

$$
f^{*}(s)=\sum_{n=0}^{+\infty} b_{n} e^{s \mu_{n}^{*}}, \quad \mu_{n}^{*}=\mu_{n}+\Delta_{n},
$$

is absolutely convergent in the whole half-plane $\Pi_{0}:=\{s: \operatorname{Re} s<0\}$ and $\sigma_{\text {abs }}\left(f^{*}\right)=0$. Indeed, for every fixed $s \in \Pi_{0}$ we have as $q=-\operatorname{Re} s$

$$
\left|b_{n} e^{s \mu_{n}^{*}}\right|=\left|\frac{b_{n}}{\alpha_{n}^{q}} e^{s \mu_{n}}\right| \quad(\forall n \geq 0) .
$$

But $\sigma_{\text {abs }}\left(f_{q}\right)=0$, thus $\sigma_{\text {abs }}\left(f^{*}\right) \geq 0$. In the other hand $\left.b_{n} e^{s \mu_{n}^{*}}\right|_{s=0}=e^{\lambda_{n}} \nrightarrow \rightarrow 0$ $(n \rightarrow+\infty)$, hence $\sigma_{\text {abs }}\left(f^{*}\right)=0$.

From condition (2) (see proof of Lemma 2 in [1, p.121-122]) we get

$$
\nu\left(x, f^{*}\right) \rightarrow+\infty \quad(x \rightarrow-0) .
$$

We need the following lemma (compare, for example, $[7,8]$ ).

Lemma 2.1. For all $n \geq 0$ and $k \geq 1$ inequality

$$
\frac{\alpha_{n}}{\alpha_{k}} e^{\tau_{k}\left(\mu_{n}-\mu_{k}\right)} \leq e^{-\delta|n-k|}
$$

holds, where $\tau_{k}:=t_{k}+\frac{\delta}{\mu_{k}-\mu_{k-1}}, t_{k}:=\frac{\Delta_{k-1}-\Delta_{k}}{\mu_{k}-\mu_{k-1}}$.

Proof of Lemma 2.1. We remark that

$$
\begin{gathered}
t_{k}=-\delta \cdot \sum_{m=k}^{+\infty}\left(\frac{1}{\mu_{m}-\mu_{m-1}}+\frac{1}{\mu_{m+1}-\mu_{m}}\right) \\
\tau_{k}=-2 \delta \cdot \sum_{m=k+1}^{+\infty} \frac{1}{\mu_{m}-\mu_{m-1}} \\
\tau_{k+1}-\tau_{k}=\frac{2 \delta}{\mu_{k+1}-\mu_{k}} \quad(k \geq 1) .
\end{gathered}
$$

Since $\ln \alpha_{n}-\ln \alpha_{n-1}=\Delta_{n}-\Delta_{n-1}=-t_{n}\left(\mu_{n}-\mu_{n-1}\right)$, for $n \geq k+1$ we have

$$
\ln \frac{\alpha_{n}}{\alpha_{k}}+\tau_{k}\left(\mu_{n}-\mu_{k}\right)=-\sum_{j=k+1}^{n} t_{j}\left(\mu_{j}-\mu_{j-1}\right)+\tau_{k} \sum_{j=k+1}^{n}\left(\mu_{j}-\mu_{j-1}\right)=
$$




$$
\begin{gathered}
=-\sum_{j=k+1}^{n}\left(t_{j}-\tau_{k}\right)\left(\mu_{j}-\mu_{j-1}\right) \leq-\sum_{j=k+1}^{n}\left(t_{j}-\tau_{j-1}\right)\left(\mu_{j}-\mu_{j-1}\right)= \\
=-\sum_{j=k+1}^{n} \delta=-(n-k) \cdot \delta .
\end{gathered}
$$

Similarly, for $n \leq k-1$ we obtain

$$
\begin{gathered}
\ln \frac{\alpha_{n}}{\alpha_{k}}+\tau_{k}\left(\mu_{n}-\mu_{k}\right)=-\ln \frac{\alpha_{k}}{\alpha_{n}}-\tau_{k}\left(\mu_{k}-\mu_{n}\right)= \\
=\sum_{j=n+1}^{k} t_{j}\left(\mu_{j}-\mu_{j-1}\right)-\tau_{k} \sum_{j=n+1}^{k}\left(\mu_{j}-\mu_{j-1}\right) \leq \\
\leq-\sum_{j=n+1}^{k}\left(\tau_{j}-t_{j}\right)\left(\mu_{j}-\mu_{j-1}\right)=-\sum_{j=n+1}^{k} \delta=-(k-n) \cdot \delta
\end{gathered}
$$

and Lemma 2.1 is proved.

We remark that from definitions of $\tau_{k}$ and $t_{k}$ (see $\left.(9),(8)\right)$ and the condition $\sum_{k=0}^{+\infty} 1 /\left(\mu_{k+1}-\mu_{k}\right)<+\infty$ it follows that there exists $k_{0}(\delta)$ such that

$$
\tau_{k} \geq-1 \quad\left(k \geq k_{0}(\delta)\right), \quad \tau_{k}<0 \quad(k \geq 1) .
$$

Let $J$ be a set of the values of the central index $\nu\left(\sigma, f^{*}\right)$, i.e.

$$
J=\left\{k \in \mathbb{N}:(\exists \sigma<0)\left[\nu\left(\sigma, f^{*}\right)=k\right]\right\} .
$$

Denote by $\left(R_{k}\right)$ a sequence of the points of the springs of $\nu\left(\sigma, f^{*}\right)$, enumerate such that $\nu\left(\sigma, f^{*}\right)=k$ for $\sigma \in\left[R_{k}, R_{k+1}\right)$ in the case $R_{k}<R_{k+1}$. Then for $\sigma \in\left[R_{k}, R_{k+1}\right), k \in J$ from Lemma 2.1 we have

$$
b_{n} e^{\sigma \mu_{n}^{*}} \leq b_{k} e^{\sigma \mu_{k}^{*}} \Longleftrightarrow \frac{b_{n} e^{\sigma \mu_{n}}}{b_{k} e^{\sigma \mu_{k}}} \leq \frac{\alpha_{n}^{|\sigma|}}{\alpha_{k}^{|\sigma|}} \leq e^{-|\sigma| \tau_{k}\left(\mu_{n}-\mu_{k}\right)} e^{-|\sigma||n-k| \cdot \delta}
$$

for all $n \geq 0$. Hence, $\frac{b_{n} e^{\left(\sigma+|\sigma| \tau_{k}\right) \mu_{n}}}{b_{k} e^{\left(\sigma+|\sigma| \tau_{k}\right) \mu_{k}}} \leq e^{-|\sigma||n-k| \cdot \delta}$ i.e., for all $k \in J$ and for every $\sigma^{*} \in\left[R_{k}\left(1+\left|\tau_{k}\right|\right), R_{k+1}\left(1+\left|\tau_{k}\right|\right)\right)$

$$
\frac{b_{n} e^{\sigma^{*} \mu_{n}}}{b_{k} e^{\sigma^{*} \mu_{k}}} \leq \exp \left\{-\frac{\left|\sigma^{*}\right||n-k| \cdot \delta}{1+\left|\tau_{k}\right|}\right\}
$$


Thus, as $x=\frac{1}{\left|\sigma^{*}\right|} \in\left[-\frac{1}{R_{k}\left(1+\left|\tau_{k}\right|\right)},-\frac{1}{R_{k+1}\left(1+\left|\tau_{k}\right|\right)}\right)$ we get

$$
\frac{\left|a_{n}\right| e^{x \lambda_{n}}}{\left|a_{k}\right| e^{x \lambda_{k}}} \leq \exp \left\{-\frac{|n-k| \cdot \delta}{1+\left|\tau_{k}\right|}\right\}
$$

for all $k \in J, n \geq 0$. Therefore,

$$
\nu(x, F)=k, \mu(x, F)=\left|a_{k}\right| e^{x \lambda_{k}}, x \in\left[-\frac{1}{R_{k}\left(1+\left|\tau_{k}\right|\right)},-\frac{1}{R_{k+1}\left(1+\left|\tau_{k}\right|\right)}\right) .
$$

Denote $E^{*}(\delta):=\bigcup_{k=k_{0}(\delta)}^{+\infty}\left[-\frac{1}{R_{k}\left(1+\left|\tau_{k}\right|\right)},-\frac{1}{R_{k+1}\left(1+\left|\tau_{k}\right|\right)}\right), E(\delta):=[0,+\infty) \backslash$ $E^{*}(\delta)$. Then for every $x>0, x \notin E(\delta)$

$$
\begin{gathered}
\mid F(x+i y)-a_{\nu(x, F)} e^{(x+i y) \lambda_{\nu(x, F)} \mid \leq} \\
\leq \mu(x, F) \cdot \sum_{n \neq \nu(x, F)} \exp \left\{-\frac{\delta \cdot|n-\nu(x, F)|}{1+\left|\tau_{\nu(x, F)}\right|}\right\} \leq \\
\leq \frac{2 e^{-\delta / 2}}{1-e^{-\delta / 2}} \cdot \mu(x, F)
\end{gathered}
$$

because $1+\left|\tau_{\nu(x, F)}\right|<2\left(x \in E^{*}(\delta)\right)$. It remains to prove that the logarithmic $h$-measure of a set $E(\delta)$ is finite. Using

$$
\begin{gathered}
E(\delta) \subset\left[0, x_{0}\right) \bigcup\left(\bigcup_{k=k_{0}(\delta)+1}^{+\infty}\left[-\frac{1}{R_{k}\left(1+\left|\tau_{k-1}\right|\right)},-\frac{1}{R_{k}\left(1+\left|\tau_{k}\right|\right)}\right)\right), \\
x_{0}=-\frac{1}{R_{k_{0}(\delta)}\left(1+\left|\tau_{k_{0}(\delta)-1}\right|\right)},
\end{gathered}
$$

and equality (10), we obtain

$$
\begin{aligned}
& \text { h-log-meas }\left(E \cap\left[x_{0},+\infty\right)\right)= \\
& =\sum_{k=k_{0}(\delta)+1}^{+\infty} \int_{\left[-\frac{1}{R_{k}\left(1+\left|\tau_{k-1}\right|\right)},-\frac{1}{R_{k}\left(1+\left|\tau_{k}\right|\right)}\right)} h(x) d \ln x \leq \\
& =\sum_{k=k_{0}(\delta)+1}^{+\infty} h\left(-\frac{1}{R_{k}\left(1+\left|\tau_{k}\right|\right)}\right) \ln \left(1+\frac{\left|\tau_{k-1}\right|-\left|\tau_{k}\right|}{1+\left|\tau_{k}\right|}\right) \leq \\
& \leq 2 \delta \cdot \sum_{k=k_{0}(\delta)}^{+\infty} h\left(-\frac{1}{R_{k+1}\left(1+\left|\tau_{k+1}\right|\right)}\right) \frac{1}{\mu_{k+1}-\mu_{k}} .
\end{aligned}
$$


The condition $F \in \mathcal{D}_{a}(\Phi)$ implies

$$
x \Phi(x) \leq \ln \mu(x, F)=-\mu_{\nu(x-0, F)}+x \lambda_{\nu(x-0, F)} \leq x \lambda_{\nu(x-0, F)}
$$

$\left(x \geq x_{1}>0\right)$, therefore

$$
x \leq \varphi\left(\lambda_{\nu(x-0, F)}\right) \quad\left(x \geq x_{1}>0\right) .
$$

Denote $\theta_{k}:=-\left(R_{k+1}\left(1+\left|\tau_{k}\right|\right)\right)^{-1}$. By (11) we have $\nu\left(\theta_{k}-0\right)=k$, thus from (10) and (14) it follows

$$
\begin{gathered}
-\frac{1}{R_{k+1}\left(1+\left|\tau_{k+1}\right|\right)}=\theta_{k} \cdot \frac{1+\left|\tau_{k}\right|}{1+\left|\tau_{k+1}\right|}=\theta_{k} \cdot\left(1+\frac{\left|\tau_{k}\right|-\left|\tau_{k+1}\right|}{1+\left|\tau_{k+1}\right|}\right) \leq \\
\leq \theta_{k} \cdot\left(1+\frac{2 \delta}{\mu_{k+1}-\mu_{k}}\right) \leq \varphi\left(\lambda_{k}\right) \cdot\left(1+\frac{2 \delta}{\mu_{k+1}-\mu_{k}}\right)
\end{gathered}
$$

for all $k \geq k_{1}(\delta)$. Using inequality (15) to inequality (13), we get

$$
\begin{gathered}
\text { h-log-meas }\left(E(\delta) \cap\left[x_{0},+\infty\right)\right) \leq \\
\leq 2 \delta \cdot \sum_{k=k_{0}(\delta)}^{k_{2}(\delta)-1} h\left(-\frac{1}{R_{k+1}\left(1+\left|\tau_{k+1}\right|\right)}\right) \frac{1}{\mu_{k+1}-\mu_{k}}+ \\
+2 \delta \cdot \sum_{k=k_{2}(\delta)}^{+\infty} h\left(\varphi\left(\lambda_{k}\right) \cdot\left(1+\frac{2 \delta}{\mu_{k+1}-\mu_{k}}\right)\right) \frac{1}{\mu_{k+1}-\mu_{k}}:=K(\delta)<+\infty,
\end{gathered}
$$

where $k_{2}(\delta)=\max \left\{k_{0}(\delta), k_{1}(\delta)\right\}$. Relation (16) implies that

$$
(\forall \delta>0): \quad \text { h-log-meas }(E(\delta) \cap[x,+\infty))=o(1) \quad(x \rightarrow+\infty) .
$$

We put now $\delta_{n}=n, \varepsilon_{n}=2^{-n}(n \geq 1)$. Then for every $n \geq 1$ there exists $x_{n} \geq$ $x_{0}$ such that h-log-meas $\left(E\left(\delta_{n}\right) \cap\left[x_{n},+\infty\right)\right) \leq \varepsilon_{n}$. Without loss of generality we may assume that $x_{n}<x_{n+1}(n \geq 1)$. Denote $E=\bigcup_{n=1}^{+\infty}\left(E\left(\delta_{n}\right) \cap\left[x_{n} ; x_{n+1}\right)\right)$. Define a function $\gamma:\left[x_{1},+\infty\right) \rightarrow[0,+\infty)$ by equality $\gamma(x)=4 / n$ for $x \in$ $\left[x_{n}, x_{n+1}\right)$. Then from inequality (12) it follows

$$
\left|F(x+i y)-a_{\nu(x, F)} e^{(x+i y) \lambda_{\nu(x, F)}}\right| \leq \gamma(x) \cdot \mu(x, F)
$$

for all $x \in\left[x_{1},+\infty\right) \backslash E$ uniformly in $y \in \mathbb{R}$ because $\gamma(x)=4 / n \geq \frac{2 e^{-\delta_{n} / 2}}{1-e^{-\delta_{n} / 2}}$ $(n \geq 1)$. But $\gamma(x) \rightarrow 0(x \rightarrow+\infty)$ and

$$
\begin{gathered}
\text { h-log-meas }\left(E \cap\left[x_{1},+\infty\right)\right) \leq \\
\leq \sum_{n=1}^{+\infty} \text { h-log-meas }\left(E\left(\delta_{n}\right) \cap\left[x_{n}, x_{n+1}\right)\right) \leq \sum_{n=1}^{+\infty} \varepsilon_{n}=1 .
\end{gathered}
$$

Thus, h-log-meas $(E)<+\infty$. 


\section{Concluding Remarks}

Since $\lambda_{n} / \mu_{n} \rightarrow 0(n \rightarrow+\infty)$, we have $\lambda_{n}<\mu_{n}$ for all $n$ large enough. So $\lambda_{n}$ one can replace with $\mu_{n}$ in (5).

In the case $\beta=\sup \left\{\lambda_{j}: j \geq 0\right\}=+\infty$ condition (5) of Theorem 1 can be written in a simpler form. For example, such theorem from Theorem 1 follows. Let $\Phi \in \mathcal{L}_{+}$and $\mathcal{D}_{a}^{*}(\Phi):=\bigcup_{\rho>0} \mathcal{D}_{a}\left(\Phi_{\rho}\right), \Phi_{\rho}(x):=\rho \cdot \Phi(x \rho)$.

Theorem 2. Let $\left(\mu_{n}\right)$ be a sequence such that condition (4) holds, $h \in$ $\mathcal{L}_{+}, \Phi \in \mathcal{L}_{+}$and $F \in \mathcal{D}_{a}^{*}(\Phi)$. If

$$
(\forall b>0): \quad \sum_{n=n_{0}}^{+\infty} \frac{h\left(b \varphi\left(b \lambda_{n}\right)\right)}{\mu_{n+1}-\mu_{n}}<+\infty,
$$

then relation (3) holds as $x \rightarrow+\infty$ outside some set $E$ of finite logarithmic $h$-measure uniformly in $y \in \mathbb{R}$.

In the case $\Phi(x)=e^{x} / x$ we obtain that $\mathcal{D}_{a}^{*}(\Phi)$ is the class Dirichlet series of nonzero lower R-order, i.e. $\underline{\lim }_{x \rightarrow+\infty} \frac{1}{x} \ln \ln M(x, F):=\rho_{R}[F] \in(0,+\infty]$ and condition (17) from condition $(\forall b>0): \sum_{n=n_{0}}^{+\infty} h\left(b \ln \lambda_{n}\right) /\left(\mu_{n+1}-\mu_{n}\right)<+\infty$ follows.

Question 3.1. Is the description of exceptional sets in our Theorems 1 and 2 the best possible?

Question 3.2. Are conditions (5) and (17) in our Theorems 1 and 2 necessary?

\section{References}

[1] O.B. Skaskiv, On the minimum of the absolute value of the sum for a Dirichlet series with bounded sequence of exponents, Math. Notes 56 (5) (1994) 1177-1184, doi: 10.1007/BF02274666.

[2] Ya. Stasyuk, On the Dirichlet series with monotonous coefficients and the finality of description of the exeptional set, Mat. Visn. Nauk. Tov. Im. Shevchenka, 5 (2008) 202-207. (in Ukrainian) http://journals.iapmm. lviv.ua/ojs/index.php/MBSSS/article/view/105/99

[3] O.B. Skaskiv, On Wiman's theorem concerning the minimum modulus of a function analytic in the unit disk, Math. USSR, Izv. 35 (1) (1990), 165-182, doi: 10.1070/IM1990v035n01ABEH000694.

[4] O.B. Skaskiv, On certain relations between the maximum modulus and the maximal term of an entire dirichlet series, Math Notes, 66 (1999) 223-232, doi: 10.1007/BF02674881.

[5] P.C. Fenton, The minimum modulus of gap power series, Proc. Edinburgh Math. Soc., 21 (1978) 49-54, doi: 10.1017/S001309150001587X. 
[6] O.B. Skaskiv, M.N. Sheremeta, On the asymptotic behavior of Dirichlet series, Mathematics of the USSR-Sbornik, 59 (1988), 379-396, doi: 10.1070/SM1988v059n02ABEH003141.

[7] M.R. Lutsyshyn, O.B. Skaskiv, Asymptotic properties of a multiple Dirichlet series, Mat. Stud., 3 (1994) 41-48. (in Ukrainian) http://matstud.org.ua/texts/1994/3/3_041-048.pdf

[8] T.M. Salo, O.B. Skaskiv, The minimum modulus of gap power series and h-measure of exceptional sets, arXiv: 1512.05557v2[math.CV] 21 Dec 2015, 13 p.

[9] S.Mandelbrojt, Dirichlet series. Principles and methods, Dordrecht: Reidel (1972). 
